\title{
ORIGINAL
}

\section{ESTUDIO BIBLIOMÉTRICO DE LOS ARTÍCULOS ORIGINALES \\ DE LA REVISTA ESPAÑOLA DE SALUD PÚBLICA (1991-2000). PARTE SEGUNDA: PRODUCTIVIDAD DE LOS AUTORES Y PROCEDENCIA INSTITUCIONAL Y GEOGRÁFICA}

José Manuel Estrada Lorenzo (1), Fernando Villar Álvarez (2), Cristina Pérez Andrés (3) y M. ' José Rebollo Rodríguez (4)

(1) Centro de Información Bibliogáfica y Documentación en Ciencias de la Salud. Agencia Laín Entralgo. Consejería de Sanidad. Madrid.

(2) Escuela Nacional de Sanidad. Instituto de Salud Carlos III. Ministerio de Sanidad y Consumo. Madrid.

(3) Comité de Redacción de la Revista Española de Salud Pública. Ministerio de Sanidad y Consumo. Madrid.

(4) Biblioteca del Colegio Oficial de Médicos. Madrid.

RESUMEN

Fundamento: En la caracterización de una revista científica, desde el punto de vista bibliométrico, es importante conocer cuántos autores participan en los trabajos publicados, así como la institución y ámbito geográfico de procedencia. El objetivo de este artículo es analizar la distribución del número de autores y de las instituciones donde éstos trabajan, así com su productividad, en los artículos originales publicados en la Revista Española de Salud Pública durante la década 1991-2000

Métodos: De los 290 trabajos originales publicados en la Revista Española de Salud Pública durante la década estudiada se ha calculado el número total de autores, el número de autores ocasionales, el índice de transitoriedad, la distribución de autores por género, la productividad de autores y de las instituciones según el número de trabajos y la de los autores según la Ley de Lotka, y la distribución por procedencia institucional y geográfica.

Resultados: En los originales que se publicaron en la Revista Española de Salud Pública entre los años 1991 y 2000 participó un total de 1.052 autores ( 1.000 españoles y 52 extranjeros). La razón de sexos para el periodo estudiado es de 1,29 a favor de los hombres. Las Comunidades Autónomas que más autores aportan son Comunidad de Madrid (16,3\%), Andalucía (13,4\%) y Comunidad Valenciana (12,5\%). Las instituciones de procedencia de los autores se encuentran ubicadas preferentemente en la Comunidad de Madric $(16,5 \%)$, Comunidad Valenciana (11,3\%), y Andalucía y Cataluña $(10,5 \%)$ El 37,6\% de los autores trabajan en centros dedicados a la atención sanitaria, seguidos de los que trabajan en la Universidad (26,3\%). Al distribuir el tipo de institución por Comunidades Autónomas, en Madrid, la Comunidad Valenciana y Andalucía la institución más frecuente son los centros de atención primaria (50\%, $43,3 \%$ y $28,6 \%$ respectivamente) y en Cataluña los cención pina cia (50\%, $43,3 \%$ y $28,6 \%$ respe

Conclusiones: La Revista Española de Salud Pública es una publicación implantada en todo el territorio nacional y en el ámbito hispanoamericano. La productividad de los autores que publican en ella, la de las instituciones donde trabajan y la de las Comunidades Autónomas de las que proceden los trabajos, es similar a la de otras revistas biomédicas españolas, y menor que cuando se estudian ámbitos temáticos y territoriales en más de una publicación.

Palabras clave: Publicaciones periódicas. Bibliometría. Índice de transitoriedad. Productividad. Revista Española de Salud Pública.

Correspondencia:

Cristina Pérez Andrés

Revista Española de Salud Pública

Ministerio de Sanidad y Consumo

Paseo del Prado, 18-20

28071 Madrid

Correo electrónico: resp@msc.es

\section{ABSTRACT}

Bibliometric study of the original articles published in the Revista Española de Salud Pública (1991-2000). Part Two: Authors, their Institutions and Geographical Areas

Background: When characterizing a scientific journal from the bibliometric standpoint, it is of importance to know how many authors were involved in the studies published as well as the geographical areas where these authors are located and the type of institutions by which they are employed. The aim of this article is that of analyzing the geographical spread of these authors and the institutions by which they are employed, as spread of these authors and the institutions by which they are employed, as
well as its evolution as regards the original articles published in the Revista Española de Salud Pública throughout the 1991-2000 period.

Methods: Of the original articles published in the Revista Española de Salud Pública throughout the studied ten-year period, a calculation has been made as to the total number of authors, the number of occasional authors (authors having published solely one article), transitivity index, the ratio between the number of male and female authors, the productivity of the authors and their institutions and the spread of authors and the institutions by which they are employed by Autonomous Communities.

Results: Of the original articles published in the Revista Española de Salud Pública throughout the 1991-2000 period, a total of 1,052 different authors were involved, 1,000 of whom were Spanish and 52 foreigners. The ratio of male authors to female authors for the period in question was 1.29 . The Autonomous Communities from which the largest number of authors The Autonomous Communities from which the largest number of authors
came were Community of Madrid (16,3\%), Andalusia $(13,4 \%)$ and the Community of Valencia $(12.5 \%)$. The institutions by which the authors are employed are located most often in Community of Madrid (16,5\%), in Community of Valencia $(11,3 \%)$ and Andalusia and Catalonia $(10,5 \%)$. A total of $37.6 \%$ of the authors work at centers devoted to health care, followed by authors who work at Universities (26.3\%). On calculating the spread of the type of institution by Autonomous Communities, in the Community of Madrid and in the Community of Valencia and Andalusia, the most frequent institution is that of the primary care centers $(50 \%, 43.3 \%$ and $28.6 \%$ respectively); and in Catalonia the specialized care centers ( $42.9 \%$ ).

Conclusions: The Revista Española de Salud Pública is a scientific journal well known in the whole spanish territory and in the Latinoamerican countries. The productivity of the authors publising in this journal, the productivity of their institutions where they work and that of Autonomou Regions where they belong to, is similar to other magazines. The produc tivity of the authors is greater when we look at other public health magazines or other countries.

Keywords: Authorship. Revista Española de Salud Pública. Periodicals. Bibliometrics. 


\section{INTRODUCCIÓN}

En este trabajo se presentan los resultados de la segunda parte del estudio bibliométrico de los originales publicados en la Revista Española de Salud Pública (RESP) durante la década 1991-2000. En el primer artículo ${ }^{1}$, dedicado a indicadores generales, se analizó el número de trabajos originales, el índice de colaboración, su distribución por Comunidad Autónoma y las materias más frecuentemente investigadas. Como se puede observar en dicho trabajo, en la década estudiada la RESP publicó 290 originales, de un total de 555 trabajos, siendo la media de 29 originales/año. El índice de colaboración en ese período fue de 4,5 y las Comunidades Autónomas que más originales publicaron fueron la Comunidad de Madrid, la Comunidad Valenciana, Andalucía y Cataluña. Entre las materias de estudio destacaron las enfermedades infecciosas y parasitarias, la atención primaria de salud y la contaminación ambiental.

De la misma forma que es relevante para la caracterización de una publicación conocer y analizar los indicadores anteriormente citados, es interesante estudiar así mismo cuál es la productividad de los autores que publican en la misma, lo que permite clasificarlos en pequeños, medianos o grandes productores ${ }^{2}$. Los mismos indicadores de productividad se pueden aplicar a las instituciones de procedencia de los autores o a las Comunidades Autónomas donde se encuentran ubicadas dichas instituciones ${ }^{2}$. Si en el estudio precedente se prestaba atención al número de firmas (un mismo autor se contabiliza como «n» firmas al haber colaborado en «n» trabajos originales), en esta parte del estudio se considera a cada autor una sola vez, para estudiar cuántos trabajos ha publicado. Así, se analiza el número total de autores que han publicado al menos una vez en RESP en el período estudiado, su procedencia geográfica por Comunidad Autónoma, y el número total de instituciones en las que trabajan los autores (teniendo en cuenta que dos o más autores de un mismo trabajo pueden pertenecer a distinta insti- tución y que un mismo autor puede tener diferentes trabajos en distintas instituciones, ya que, a lo largo de los 10 años estudiados ha podido cambiar de centro de trabajo). El cálculo del número de trabajos de cada autor y de cada institución permite determinar su productividad, clasificarlos de acuerdo a la misma, y comprobar si en la distribución de los autores por número de trabajos se cumple la ley de Lotka ${ }^{3}$. Sin embargo, como se insistía en el primer artículo, los indicadores bibliométricos no deben servir para extraer juicios de valor ni sobre autores concretos ni sobre Comunidades ni instituciones determinadas, ya que deben utilizarse como parte de un análisis más integral de cada una de estas unidades ${ }^{4}$.

Los indicadores bibliométricos de productividad pueden ser utilizados para caracterizar la producción científica de publicaciones periódicas especializadas ${ }^{5}$, de colectivos profesionales ${ }^{6}$, de ámbitos de investigación ${ }^{7}$ o de todo un país ${ }^{8}$. El trabajo que sobre la Revista de Sanidad e Higiene Pública publicó Parra en $1983^{9}$ ya comprendía un estudio sobre la productividad de los autores, pero en ese caso no se analizó su distribución geográfica ni se emprendió el análisis de las instituciones de procedencia de los trabajos publicados en la revista entre 1926 y 1975.

El objetivo de este segundo artículo es, pues, analizar la evolución y distribución cronológica, desde 1991 a 2000, del número de los autores de los trabajos originales y de sus instituciones de procedencia, distribuyéndolos de acuerdo a diversos criterios como productividad, sexo y distribución geográfica (Comunidades Autónomas) e institucional (tipo de centro de procedencia).

\section{MATERIAL Y MÉTODO}

Material de estudio: Artículos originales publicados en la RESP desde enero de 1991 hasta diciembre del año 2000, ambos inclu- 
sive, es decir, trabajos originales que aportan resultados propios, que no han sido publicados con anterioridad y que además han sido sometidos a un proceso de evaluación externa por pares para evaluar su calidad científica. Se han excluido los originales que forman parte de números monográficos, las revisiones, los editoriales, las cartas al director, las recensiones y reseñas, y las notas informativas. La inclusión de estos trabajos no originales podría contribuir a introducir algún sesgo en la selección del material de estudio.

Recogida de datos: De cada trabajo original se ha extraído la siguiente información: nombre (de 1991 a 1993 cuando éste constaba y desde 1994 en todos los artículos) y apellidos de cada autor, institución donde desarrolla su trabajo y Comunidad Autónoma o país (en el caso de los autores extranjeros) donde se ubica la institución de procedencia.

Las instituciones se han clasificado conforme a las siguientes categorías:

- Administración: instituciones dedicadas a la gestión y administración pública, divididas en ámbito nacional, autonómico y municipal.

- Atención sanitaria: instituciones dedicadas a la asistencia sanitaria, tanto especializada (hospitales y ambulatorios) como primaria (centros de salud).

- Docencia e investigación: centros docentes universitarios (facultades y escuelas universitarias), centros docentes no universitarios (escuelas e institutos de salud pública) y centros no docentes dedicados exclusivamente a la investigación.

- Otras: centros penitenciarios, organizaciones no gubernamentales, organismos internacionales o empresas privadas.
Indicadores: Se han considerado para este análisis bibliométrico los siguientes indicadores: número de autores, número de autores ocasionales (autores con un único trabajo), índice de transitoriedad (porcentaje de autores ocasionales respecto al conjunto de los autores), número de trabajos por autor y por institución, productividad de los autores según la ley de Lotka (de acuerdo a la fórmula $A n=a_{1} / n^{2}$ : el número de autores que publican «n» trabajos es inversamente proporcional al número de trabajos al cuadrado), nivel de productividad de los autores y de las instituciones, y distribución de autores e instituciones de procedencia por sexo, Comunidad Autónoma y tipo de centro.

Análisis de los datos: Para la introducción y análisis de los datos se ha utilizado el programa Excel. Para el control de calidad de la información ha sido realizada una doble entrada de datos, corrigiendo con los artículos originales los posibles errores.

\section{RESULTADOS}

El número total de autores que han publicado trabajos originales en la RESP entre 1991 y 2000 es de 1.052 (tabla 1), con un rango de 73 en 1996 a 171 en 1998. La media anual es de 105,2 autores/año. El número de autores nuevos fue mínimo en el año 1996 (64) y máximo en 1991 (165) y en 1998 (142). El incremento porcentual de autores nuevos desciende cronológicamente desde el 54,5\% del año 1992 al 8,5\% de 1999. Las cifras anuales de autores ocasionales (los que han publicado un solo trabajo) están bastante próximas a las cifras anuales totales de autores, e incluso en algunos años, como 1996 y 1997, son idénticas (73 y 177 autores, respectivamente), obteniéndose una media de anual de 118,3 autores ocasionales. Esta proximidad se observa con mayor claridad al analizar el índice de transitoriedad (porcentaje de autores ocasionales en el conjunto de autores), cuyo valor en cada año oscila entre el $89,1 \%$ de 1991 y el $100 \%$ de 1996 o 1997 (con una media de 96,1\%). 
Tabla 1

Evolución del número de autores

\begin{tabular}{|cccccc|}
\hline Año & \multicolumn{3}{c}{$\begin{array}{c}\text { Autores } \\
\text { nuevos }\end{array}$} & & \multicolumn{2}{c|}{$\begin{array}{c}\text { Índice de } \\
\text { transitoriedad }\end{array}$} \\
\cline { 2 - 6 } & $\mathbf{n}$ & $\mathbf{( \% )}$ & acumulado & anual & acumulado \\
\hline $\mathbf{1 9 9 1}$ & 165 & $(100,0)$ & 165 & 89,1 & 89,1 \\
$\mathbf{1 9 9 2}$ & 90 & $(54,5)$ & 255 & 93,6 & 87,8 \\
$\mathbf{1 9 9 3}$ & 98 & $(38,4)$ & 353 & 93,8 & 87,5 \\
$\mathbf{1 9 9 4}$ & 125 & $(35,4)$ & 478 & 95,2 & 86,6 \\
$\mathbf{1 9 9 5}$ & 94 & $(19,7)$ & 572 & 98,3 & 85,5 \\
$\mathbf{1 9 9 6}$ & 64 & $(11,2)$ & 636 & 100,0 & 85,5 \\
$\mathbf{1 9 9 7}$ & 102 & $(16,0)$ & 738 & 100,0 & 86,2 \\
$\mathbf{1 9 9 8}$ & 142 & $(19,2)$ & 880 & 95,9 & 85,9 \\
$\mathbf{1 9 9 9}$ & 75 & $(8,5)$ & 955 & 98,1 & 84,6 \\
$\mathbf{2 0 0 0}$ & 97 & $(10,2)$ & 1.052 & 97,4 & 84,8 \\
\hline Media & & $(31,3)$ & & 96,1 & \\
\hline
\end{tabular}

Los datos acumulados para la década revelan que hay 892 autores ocasionales (de 1.052 autores totales), lo que equivale a un índice de transitoriedad del 84,8\%.

Como puede apreciarse en la figura 1, si bien el número de autores nuevos se ha ido manteniendo más o menos constante durante toda la década, la tendencia del número total de autores y la del número de autores ocasionales muestran un carácter ascendente a lo largo del período y, corroborando los datos porcentuales comentados anteriormente, continúan próximos al final de la década.

Como reflejaban los datos de autores ocasionales y el índice de transitoriedad, el por-

Figura 1

Evolución cronológica del número de autores de originales en la RESP (1991-2000)

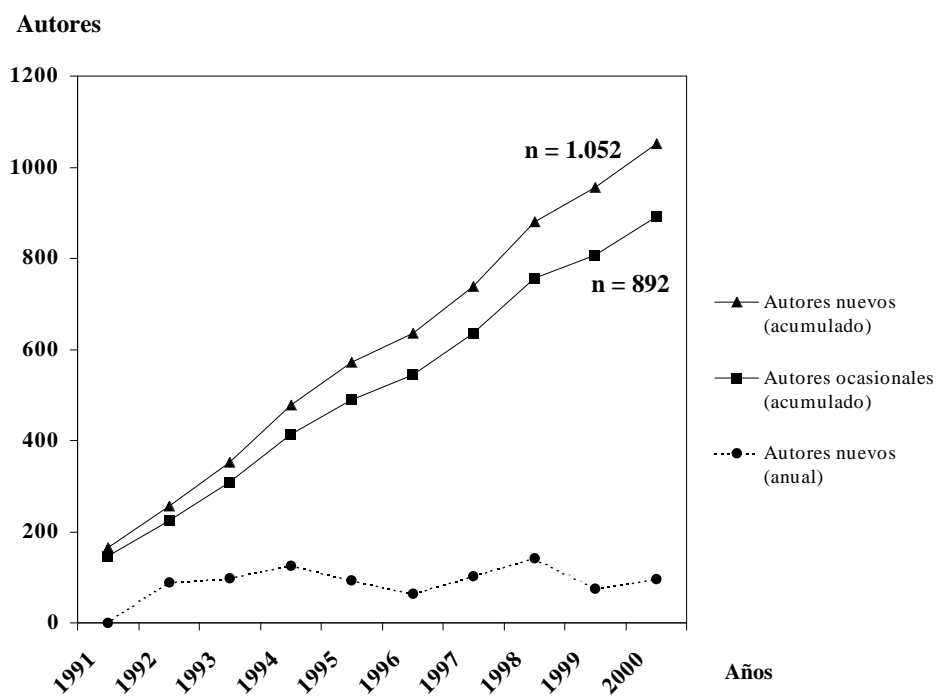


Tabla 2

Distribución cronológica del número de autores según el número de originales

\begin{tabular}{|c|c|c|c|c|c|c|c|c|}
\hline \multirow[t]{2}{*}{ Peńodo } & \multicolumn{7}{|c|}{$\begin{array}{c}\text { Autores con } \\
\text { "n" trabajos originales }\end{array}$} & \multirow[t]{2}{*}{ Total } \\
\hline & 1 & 2 & 3 & 4 & 5 & 6 & 7 & \\
\hline 1991 & 147 & 16 & 2 & -- & -- & -- & -- & 165 \\
\hline 1991-1992 & 224 & 15 & 14 & 2 & -- & -- & -- & 255 \\
\hline 1991-1993 & 309 & 24 & 13 & 4 & 1 & 2 & -- & 353 \\
\hline 1991-1994 & 412 & 43 & 15 & 4 & 1 & 2 & 1 & 478 \\
\hline 1991-1995 & 487 & 55 & 21 & 5 & 1 & 2 & 1 & 572 \\
\hline 1991-1996 & 544 & 62 & 20 & 5 & 2 & 2 & 1 & 636 \\
\hline 1991-1997 & 636 & 69 & 22 & 6 & 2 & 2 & 1 & 738 \\
\hline $1991-1998$ & 756 & 85 & 24 & 10 & 1 & 3 & 1 & 880 \\
\hline 1991-1999 & 808 & 100 & 29 & 10 & 4 & 2 & 2 & 955 \\
\hline $1991-2000$ & 892 & 108 & 32 & 12 & 4 & 2 & 2 & 1.052 \\
\hline $1991-2000$ & $(84,8 \%)$ & $(10,3 \%)$ & $(3,0 \%)$ & $(1,1 \%)$ & $(0,4 \%)$ & $(0,2 \%)$ & $(0,2 \%)$ & $(100 \%)$ \\
\hline
\end{tabular}

centaje de autores que en los distintos períodos han publicado un único trabajo es mayoritario $(84,8 \%)$ (tabla 2$)$. Asimismo se aprecia que desde el primer año hay autores que participan en 2 ó 3 trabajos, aunque los porcentajes para toda la década son: $10,3 \%$ de autores con 2 trabajos y $3 \%$ con 3 trabajos. El porcentaje de autores que a lo largo de los diez años han colaborado en más de 3 traba- jos representa el 1,9\%. Dos autores han participado en 7 trabajos originales $(0,2 \%)$, siendo este número el máximo de trabajos realizados por un autor.

En la figura 2 puede apreciarse el resultado de la aplicación de la Ley de Lotka a la productividad de los autores que han publicado en la RESP durante los años 1991-

Figura 2

Aplicación de la Ley de Lotka a la productividad de los autores de los originales de la RESP (1991-2000)

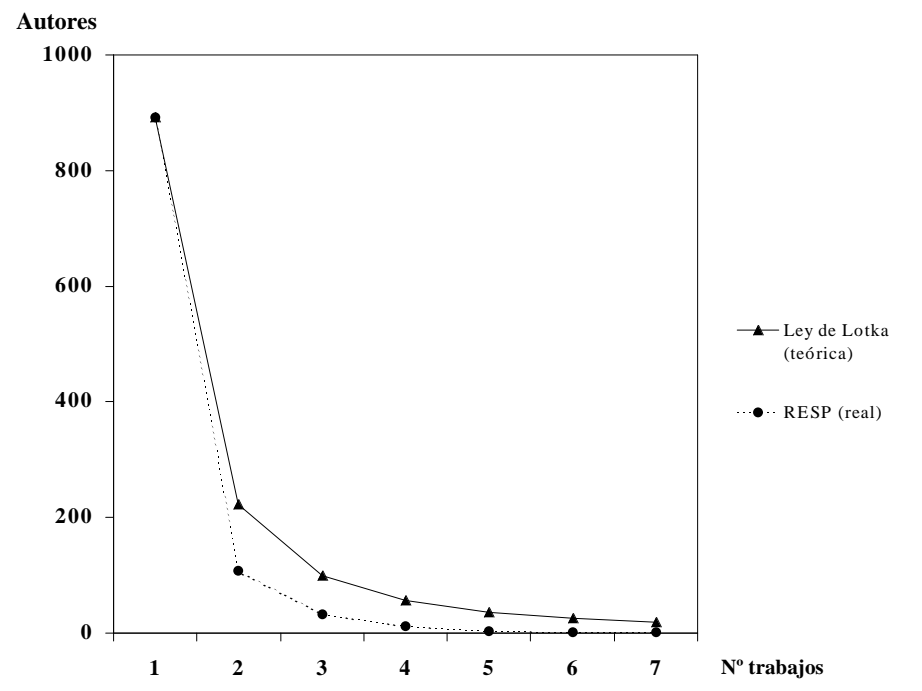


2000. La curva resultante es similar a la teórica, si bien la real es más corta y está más cercana al eje de abscisas.

En la tabla 3 puede observarse la distribución de los autores y las instituciones según nivel de productividad. El número máximo de trabajos publicados por una misma institución es de 12, caso que se da en el Instituto de Salud Carlos III (Madrid) y en la Universidad de Valencia (tabla 4) (figura 3).

La distribución de los autores por género (tabla 5) permite apreciar cómo en el periodo 1994-2000 la razón entre hombres y mujeres es siempre favorable a los primeros, excepto en el año 1996, en que esta razón se invierte a favor de las autoras $(n=39)$. La razón por sexos es máxima en el año $1997(1,78)$, siendo la media para todo el periodo de 1,29. Entre el total de los 290 originales en los que figura el nombre de pila, hay 91 mujeres que figuran como primeras autoras, por lo que se puede decir que al menos en el 31,4\% de los trabajos hay una mujer como autora principal.

Al estudiar la distribución de los autores por CCAA (tabla 6), los valores máximos corresponden a Comunidad de Madrid: 172 (16,3\%), Andalucía: $141(13,4 \%)$ y Comunidad Valenciana: 131 (12,5\%). Entre estas tres comunidades suman 444 autores, lo que equivale al 44,4\% de los 1.000 autores considerados en la totalidad del territorio español. La ubicación más frecuente de las insti-

Tabla 3

Distribución de autores e instituciones por nivel de productividad

\begin{tabular}{|cccccc|}
\hline $\begin{array}{c}\text { Nivel de } \\
\text { productividad }\end{array}$ & $\mathbf{N}^{\mathbf{0}}$ de trabajos & \multicolumn{2}{c}{ Autores } & \multicolumn{2}{c|}{ Instituciones } \\
\cline { 2 - 6 } & & $\mathbf{n}$ & $\mathbf{( \% )}$ & $\mathbf{n}$ & $\mathbf{( \% )}$ \\
\hline Grandes productores & 10 ó más & 0 & $(0,0)$ & 7 & $(2,6)$ \\
Medianos productores & $2-9$ & 160 & $(15,2)$ & 69 & $(26,0)$ \\
Pequeños productores & 1 & 892 & $(84,8)$ & 190 & $(71,4)$ \\
\hline Total & & 1.052 & $(100,0)$ & 266 & $(100,0)$ \\
\hline
\end{tabular}

Tabla 4

Instituciones con mayor productividad (8 trabajos o más)

\begin{tabular}{|lc|}
\hline Institución & $\begin{array}{c}\text { Número de trabajos } \\
\text { (\%) }\end{array}$ \\
\hline Instituto de Salud Carlos III & $12(4,1 \%)$ \\
Universidad de Valencia & $12(4,1 \%)$ \\
Instituto Municipal de la Salud & $10(3,4 \%)$ \\
Consejería Sanidad de Andalucía & $10(3,4 \%)$ \\
Universidad de Oviedo & $10(3,4 \%)$ \\
Universidad de Cádiz & $10(3,4 \%)$ \\
Consejería Sanidad de la Comunidad Valenciana & $10(3,4 \%)$ \\
Universidad de Granada & $9(3,1 \%)$ \\
Consejería Sanidad de Castilla-La Mancha & $8(2,7 \%)$ \\
Instituto Valenciano de Estudios en Salud Pública & $8(2,7 \%)$ \\
Departamento Sanidad de Cataluña & $8(2,7 \%)$ \\
\hline Total & $107(36,9 \%)$ \\
\hline
\end{tabular}


Figura 3

Distribución de instituciones por número de originales (1991-2000)

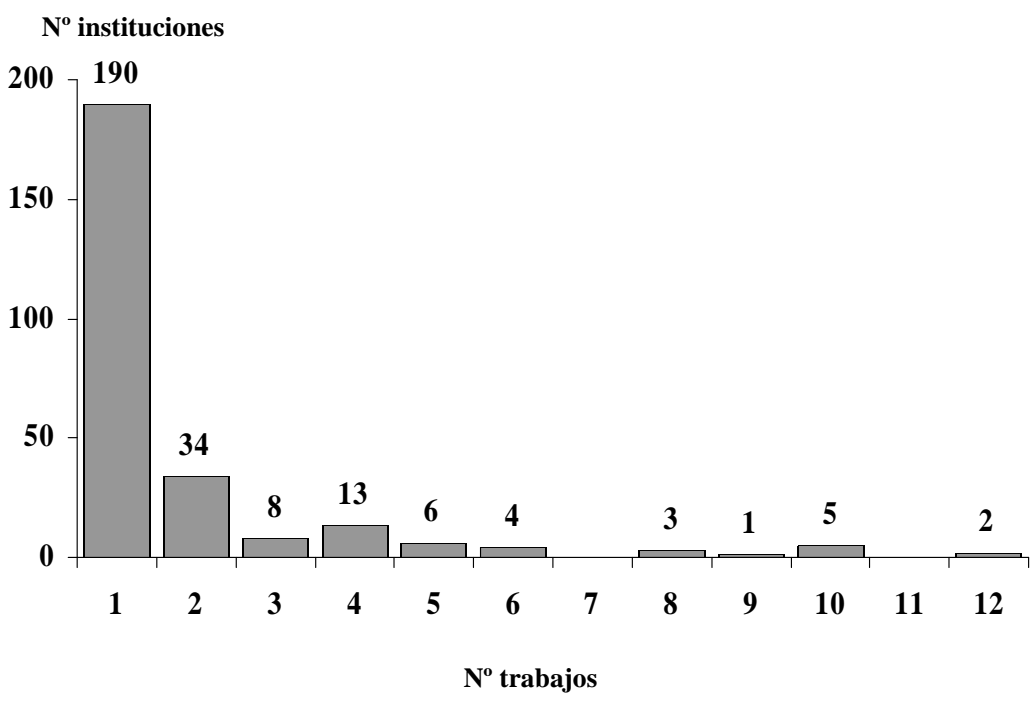

Tabla 5

Distribución anual de autores según género (1994-2000)*

\begin{tabular}{|ccccccc|}
\hline Año & $\begin{array}{c}\text { Total } \\
\text { autores }\end{array}$ & \multicolumn{2}{c}{$\begin{array}{c}\text { Hombres } \\
\text { (a) }\end{array}$} & \multicolumn{2}{c}{$\begin{array}{c}\text { Mujeres } \\
\text { (b) }\end{array}$} & (a) / (b) \\
\hline & $\mathbf{n}$ & $\mathbf{n}$ & $\mathbf{( \% )}$ & $\mathbf{n}$ & $\mathbf{\%}$ & \\
\cline { 2 - 7 } $\mathbf{1 9 9 4}$ & 147 & 88 & $(59,9)$ & 59 & $(40,1)$ & 1,49 \\
$\mathbf{1 9 9 5}$ & 119 & 60 & $(50,4)$ & 59 & $(49,6)$ & 1,01 \\
$\mathbf{1 9 9 6}$ & 73 & 34 & $(46,6)$ & 39 & $(53,4)$ & 0,87 \\
$\mathbf{1 9 9 7}$ & 117 & 75 & $(64,1)$ & 42 & $(35,9)$ & 1,78 \\
$\mathbf{1 9 9 8}$ & 171 & 99 & $(57,9)$ & 72 & $(42,1)$ & 1,37 \\
$\mathbf{1 9 9 9}$ & 107 & 60 & $(56,1)$ & 47 & $(43,9)$ & 1,27 \\
$\mathbf{2 0 0 0}$ & 114 & 63 & $(55,3)$ & 51 & $(44,7)$ & 1,23 \\
\hline Total & 848 & 479 & $(56,5)$ & 369 & $(43,5)$ & 1,29 \\
\hline
\end{tabular}

*Entre 1991 y 1993 los autores firmaban los artículos indicando apellidos e inicial del nombre. Por ello, no ha sido posible identificar en todos los casos el género y se ha optado por prescindir de la información relativa a esos años.

tuciones de procedencia de los autores por CCAA son Comunidad de Madrid con 44 instituciones (16,5\%), Comunidad Valenciana con $30(11,3 \%)$ y Andalucía y Cataluña, ambas con 28 instituciones cada una $(10,5 \%)$, correspondiendo a estas cuatro CCAA un total de 130 instituciones, lo que supone el $52,2 \%$ de las 249 instituciones ubicadas en España.
Del extranjero proceden 52 autores $(4,9 \%)$ que trabajan en 17 instituciones diferentes $(6,4 \%)$. La tabla 7 muestra con mayor detalle la distribución de los autores e instituciones en las que trabajan, los cuales se reparten principalmente entre países de habla hispana como Cuba, Argentina y México. Cabe destacar que de los 18 trabajos en que participan autores extranjeros, en 
Tabla 6

Distribución de número de autores y número de instituciones de procedencia por Comunidad Autónoma

\begin{tabular}{|lcccc|}
\hline \multicolumn{1}{c}{$\begin{array}{c}\text { Comunidad } \\
\text { Autónoma }\end{array}$} & & Autores & \multicolumn{2}{c|}{$\begin{array}{c}\text { Instituciones de } \\
\text { procedencia }\end{array}$} \\
\hline & $\mathbf{n}$ & $\mathbf{( \% )}$ & $\mathbf{n}$ & $\mathbf{( \% )}$ \\
Madrid & 172 & $(16,3)$ & 44 & $(16,5)$ \\
Andalucía & 141 & $(13,4)$ & 28 & $(10,5)$ \\
Com. Valenciana & 131 & $(12,5)$ & 30 & $(11,3)$ \\
Castilla y León & 92 & $(8,7)$ & 19 & $(7,1)$ \\
Cataluña & 86 & $(8,2)$ & 28 & $(10,5)$ \\
Castilla-La Mancha & 54 & $(5,1)$ & 17 & $(6,4)$ \\
Asturias & 52 & $(4,9)$ & 14 & $(5,3)$ \\
Murcia & 45 & $(4,3)$ & 9 & $(3,4)$ \\
Extremadura & 42 & $(4,0)$ & 7 & $(2,6)$ \\
Aragón & 41 & $(3,9)$ & 11 & $(4,1)$ \\
Canarias & 37 & $(3,5)$ & 7 & $(2,6)$ \\
Galicia & 32 & $(3,0)$ & 10 & $(3,8)$ \\
País Vasco & 22 & $(2,1)$ & 7 & $(2,6)$ \\
Cantabria & 21 & $(2,0)$ & 4 & $(1,5)$ \\
Baleares & 16 & $(1,5)$ & 7 & $(2,6)$ \\
Navarra & 16 & $(1,5)$ & 7 & $(2,6)$ \\
La Rioja & 0 & $(0)$ & 0 & $(0)$ \\
Total CC.AA. & 1.000 & $(95,1)$ & 249 & $(93,6)$ \\
Extranjero & 52 & $(4,9)$ & 17 & $(6,4)$ \\
\hline Total & 1.052 & $(100,0)$ & 266 & $(100,0)$ \\
\hline
\end{tabular}

Tabla 7

Distribución de los países extranjeros por número de trabajos originales, autores e instituciones de procedencia

\begin{tabular}{|lccc|}
\hline \multicolumn{1}{|c}{ País } & $\begin{array}{c}\mathbf{N}^{\mathbf{0}} \\
\text { trabajos }\end{array}$ & $\begin{array}{c}\mathbf{N}^{\mathbf{0}} \\
\text { autores }\end{array}$ & $\begin{array}{c}\mathbf{N}^{\mathbf{0}} \\
\text { instituciones }\end{array}$ \\
\hline Alemania & $1^{*}$ & 1 & 1 \\
Argentina & 4 & 13 & 4 \\
Cuba & 4 & 18 & 3 \\
Estados Unidos & 1 & 2 & 1 \\
México & 3 & 9 & 5 \\
Países Bajos & $2^{*}$ & 4 & 1 \\
Portugal & $1^{*}$ & 1 & 1 \\
Reino Unido & $2^{*}$ & 4 & 1 \\
\hline Total & $18^{* *}$ & 52 & 17 \\
\hline
\end{tabular}

* Trabajos realizados en colaboración con autores e instituciones españoles.

**La suma de los trabajos supera el total de 18 porque varios países colaboran en los mismos trabajos.

5 de ellos han colaborado con autores e instituciones españoles.
La distribución de los autores por tipo de institución de procedencia se recoge en la tabla 
8. En ella se refleja cómo los autores de los artículos estudiados trabajan, sobre todo, en atención sanitaria (37,6\%). Detrás se sitúan las instituciones dedicadas a la docencia y la investigación $(32,4 \%)$ y las de la administración $(24,4 \%)$. No obstante, al desagregar por categorías el mayor número de autores trabaja en la Universidad (26,3\%), seguidos de los que trabajan en instituciones de atención especializada $(20,2 \%)$ y atención primaria $(17,4 \%)$.
La distribución de las instituciones por tipo de centro (tabla 9) permite observar cómo también las instituciones de atención sanitaria son las más frecuentes $(58,3 \%)$, seguidas de las instituciones docentes y de investigación $(19,5 \%)$ y en tercer lugar las de la administración $(14,7 \%)$. En cambio, en este caso, al desagregar por categorías el tipo de institución más frecuente son los centros de atención primaria $(31,2 \%)$ seguidos de

Tabla 8

Distribución de los autores por tipo de institución de procedencia

\begin{tabular}{|llrr|}
\hline \multicolumn{1}{c}{$\begin{array}{c}\text { Tipo de } \\
\text { Institución }\end{array}$} & \multicolumn{1}{c|}{ Nivel } & Autores \\
\cline { 3 - 4 } & & $\mathbf{N}$ & $\mathbf{( \% )}$ \\
\hline Administración & & 60 & $(5,7)$ \\
$257(24,4 \%)$ & Ámbito nacional & 173 & $(16,4)$ \\
& Ámbito autonómico & 24 & $(2,3)$ \\
\hline Atención sanitaria & Ámbito municipal & 213 & $(10,2)$ \\
$396(37,6 \%)$ & Atención especializada & 183 & $(26,3)$ \\
\hline Docencia e investigación & Atención primaria & 277 & $(2,9)$ \\
$341(32,4 \%)$ & Universitario & 30 & $(3,2)$ \\
\hline Otros & No universitario & 34 & $(2,2)$ \\
$43(4,1 \%)$ & Investigación & 11 & $(1,0)$ \\
& Penitenciario & 9 & $(0,9)$ \\
\hline No consta en trabajo original & ONG & & $(1,4)$ \\
$15(1,4 \%)$ & Varios & 15 & $\mathbf{( 1 0 0 )}$ \\
\hline Total & & $\mathbf{1 . 0 5 2}$ & \\
\hline
\end{tabular}

Tabla 9

Distribución de las instituciones por tipo y ámbito

\begin{tabular}{|llcc|}
\hline \multirow{2}{*}{$\begin{array}{c}\text { Tipo de } \\
\text { Institución }\end{array}$} & Nivel & \multicolumn{2}{c|}{ Instituciones } \\
\cline { 3 - 4 } & & $\mathbf{n}$ & $\mathbf{( \% )}$ \\
\hline Administración & Ámbito nacional & 14 & $(5,3)$ \\
$39(14,7 \%)$ & Ámbito autonómico & 20 & $(7,5)$ \\
& Ámbito municipal & 5 & $(1,9)$ \\
\hline Atención sanitaria & Atención especializada & 72 & $(27,1)$ \\
$155(58,3 \%)$ & Atención primaria & 83 & $(31,2)$ \\
\hline Docencia e investigación & Univer sitario & 37 & $(13,9)$ \\
$52(19,5 \%)$ & No universitario & 7 & $(2,6)$ \\
& Investigación & 8 & $(3,0)$ \\
\hline Otros & Penitenciario & 12 & $(4,5)$ \\
$20(7,5 \%)$ & ONG & 4 & $(1,5)$ \\
& Varios & 4 & $(1,5)$ \\
\hline Total & & $\mathbf{2 6 6}$ & $\mathbf{( 1 0 0 )}$ \\
\hline
\end{tabular}


Tabla 10

Distribución del tipo de institución por Comunidad Autónoma

\begin{tabular}{|c|c|c|c|c|c|c|c|c|c|c|c|}
\hline \multirow{3}{*}{ Comunidad Autónoma } & & & \multicolumn{4}{|c|}{ Atención sanitaria } & \multirow{2}{*}{\multicolumn{2}{|c|}{$\begin{array}{c}\text { Docencia } \\
\mathbf{e} \\
\text { investigación }\end{array}$}} & \multirow{2}{*}{\multicolumn{2}{|c|}{ Otros }} & \multirow{3}{*}{$\begin{array}{r}\text { Total } \\
n\end{array}$} \\
\hline & \multicolumn{2}{|c|}{ Administración } & \multicolumn{2}{|c|}{ Especializada } & \multicolumn{2}{|c|}{ Primaria } & & & & & \\
\hline & $\mathbf{n}$ & $(\%)$ & $\mathbf{n}$ & (\%) & $\mathbf{n}$ & (\%) & $\mathbf{n}$ & $(\%)$ & $\mathbf{n}$ & $(\%)$ & \\
\hline Andalucía & 4 & $(14,3)$ & 7 & $(25,0)$ & 8 & $(28,6)$ & 6 & $(21,4)$ & 3 & $(10,7)$ & 28 \\
\hline Aragón & 2 & $(18,2)$ & 2 & $(18,2)$ & 5 & $(45,5)$ & 2 & $(18,2)$ & 0 & 0 & 11 \\
\hline Asturias & 4 & $(28,6)$ & 3 & $(21,4)$ & 6 & $(42,9)$ & 1 & $(7,1)$ & 0 & 0 & 14 \\
\hline Baleares & 1 & $(14,3)$ & 2 & $(28,6)$ & 3 & $(42,9)$ & 1 & $(14,3)$ & 0 & 0 & 7 \\
\hline Canarias & 1 & $(14,3)$ & 3 & $(42,9)$ & 0 & 0 & 2 & $(28,6)$ & 1 & $(14,3)$ & 7 \\
\hline Cantabria & 1 & $(25,0)$ & 0 & 0 & 1 & $(25,0)$ & 1 & $(25,0)$ & 1 & $(25,0)$ & 4 \\
\hline Cataluña & 4 & $(14,3)$ & 12 & $(42,9)$ & 2 & $(7,1)$ & 7 & $(25,0)$ & 3 & $(10,7)$ & 28 \\
\hline Castilla y León & 1 & $(5,3)$ & 8 & $(42,1)$ & 5 & $(26,3)$ & 3 & $(15,8)$ & 2 & $(10,5)$ & 19 \\
\hline Castilla-La Mancha & 2 & $(11,8)$ & 3 & $(17,6)$ & 9 & $(52,9)$ & 1 & $(5,9)$ & 2 & $(11,8)$ & 17 \\
\hline Extremadura & 1 & $(14,3)$ & 2 & $(28,6)$ & 3 & $(42,9)$ & 1 & $(14,3)$ & 0 & $(0)$ & 7 \\
\hline Galicia & 1 & $(10,0)$ & 2 & $(20,0)$ & 2 & $(20,0)$ & 2 & $(20,0)$ & 3 & $(30,0)$ & 10 \\
\hline Madrid & 6 & $(13,6)$ & 9 & $(20,5)$ & 22 & $(50,0)$ & 5 & $(11,4)$ & 2 & $(4,5)$ & 44 \\
\hline Murcia & 1 & $(11,1)$ & 4 & $(44,4)$ & 3 & $(33,3)$ & 1 & $(11,1)$ & 0 & (0) & 9 \\
\hline Navarra & 3 & $(42,9)$ & 2 & $(28,6)$ & 1 & $(14,3)$ & 1 & $(14,3)$ & 0 & (0) & 7 \\
\hline Com. Valenciana & 2 & $(6,7)$ & 9 & $(30,0)$ & 13 & $(43,3)$ & 6 & $(20,0)$ & 0 & (0) & 30 \\
\hline La Rioja & 0 & (0) & 0 & (0) & 0 & 0 & 0 & (0) & 0 & (0) & 0 \\
\hline País Vasco & 1 & $(14,3)$ & 2 & $(28,6)$ & 0 & 0 & 2 & $(28,6)$ & 2 & $(28,6)$ & 7 \\
\hline Total & 35 & $(14,1)$ & 70 & $(28,1)$ & 83 & $(33,3)$ & 42 & $(16,9)$ & 19 & $(7,6)$ & 249 \\
\hline Extranjero & 4 & $(23,5)$ & 2 & $(11,8)$ & 0 & 0 & 10 & $(58,8)$ & 1 & $(5,9)$ & 17 \\
\hline Total & 39 & $(14,7)$ & 72 & $(27,1)$ & 83 & $(31,2)$ & 52 & $(19,5)$ & 20 & $(7,5)$ & 266 \\
\hline
\end{tabular}

los de atención especializada $(27,1 \%)$ y, a mayor distancia, figuran los centros docentes universitarios $(13,9 \%)$.

En la tabla 10 se muestra la distribución del tipo de institución por CCAA. En ella se puede observar que los autores de la Comunidad de Madrid, Comunidad Valenciana y Andalucía trabajan mayoritariamente en centros de atención primaria, y los de Cataluña en centros de atención especializada. En el caso de las instituciones extranjeras $(n=17)$, éstas se reparten principalmente entre instituciones docentes y de investigación $(58,8 \%)$ e instituciones de la administración $(23,5 \%)$.

\section{DISCUSIÓN}

Como se había indicado en la primera parte de este estudio, en la RESP se habían contabilizado 1.295 firmas en el conjunto de los 290 originales en el periodo 1991-2000. Estas 1.295 firmas proceden de un total de 1.052 autores, cifras en principio bastante cercanas, lo que parece indicar dos cosas: una baja productividad de los autores y un grado considerable de colaboración entre ellos $(4,5)^{1}$. Por otro lado, anualmente han participado una media de 105,2 autores, cifra que puede considerarse importante si se tiene en cuenta que se trata de una revista especializada. Si se compara con años anteriores, en la entonces Revista de Sanidad e Higiene Pública, entre 1926 y 1975 habían publicado un total de 932 autores $^{9}$, de lo que se deriva una media anual de 18,6. Esto es, en los primeros cincuenta años de la revista publicaron 100 autores menos que en la década 1991-2000, habiéndose casi sextuplicado la media anual de autores. Ello indica que la participación de los autores en la RESP se ha ido incrementando notablemente.

El índice de transitoriedad para el periodo es algo elevado $(84,8)$, ya que aunque desciende a lo largo de la década (en 1991 era de $89,1)$, se mantiene alto. Aunque de ello podría interpretarse, como ya se ha comentado, una baja productividad, parece intere- 
sante resaltar otro aspecto, que sería el carácter poco endogámico de la revista, ya que no se repiten sistemáticamente ni los mismos autores ni los mismos grupos. Por otra parte, la RESP no es la única revista española en el área de la salud pública y la administración sanitaria, por lo que para conocer la productividad de sus autores habría que estudiar el total de sus publicaciones independientemente de la revista en la que se encuentran publicadas.

En el estudio bibliométrico de Parra ${ }^{9}$, de los 932 autores el $83 \%$ había publicado un solo trabajo (porcentaje muy similar al que ha mostrado la revista entre los años 1991 y 2000). Si bien un solo autor había llegado a publicar 36 trabajos, en el presente estudio es mucho menor la productividad máxima de los autores, ya que sólo 2 de ellos han participado en 7 trabajos originales. Un caso similar a la RESP lo ofrece la revista Mapfre $^{10}$ para el mismo período, pues su índice de transitoriedad es de 85,5. Sin embargo, en otros trabajos donde se analiza la productividad no en una sola revista sino en un área temática determinada, el índice de transitoriedad suele ser menor. Así, en un trabajo dedicado a la producción científica en epidemiología y salud pública en diferentes revistas en el quinquenio 1988-19927 , y en el dedicado a los autores españoles que publican trabajos de neurociencia en revistas extranjeras ${ }^{11}$, el índice de transitoriedad se mantenía en 82,5 . Y en otro trabajo similar dedicado a la atención primaria en el mismo período 1988-1992 y también en revistas distintas $^{12}$, el índice de transitoriedad se establecía en 80,9. Esto es, el índice de transitoriedad suele ser más elevado cuando el análisis se centra en una sola publicación que cuando se amplía a un campo temático, lo que permite ver que los autores difunden los resultados de sus investigaciones en diferentes publicaciones, aumentando con ello su índice de productividad y disminuyendo, lógicamente, el índice de transitoriedad. Por otra parte, el haber estudiado en la RESP solamente los artículos originales, contribu- ye también a bajar la productividad de los autores y a subir, por lo tanto, el índice de transitoriedad.

Del índice de transitoriedad alto se deriva que la mayoría de los autores de la RESP se encuadran en el grupo de pequeños productores (un único trabajo) y que no existe ninguno dentro del grupo de grandes productores (10 ó más trabajos). Cifras similares ofrece el estudio de la revista Mapfre, cuyo índice de transitoriedad es similar al de RESP, donde el 14,6\% de los autores se consideran medianos productores (en RESP son el $15,2 \%$ ) y tampoco hay ninguno autor dentro del grupo de los grandes productores ${ }^{10}$. En los estudios temáticos mencionados tampoco difieren mucho los porcentajes: así, para el estudio sobre epidemiología y salud pública, el 17,3\% de los autores se clasifica como medianos productores y el $0,2 \%$ como grandes productores ${ }^{7}$; y para el de atención primaria, el $18,9 \%$ son medianos productores y el $0,2 \%$, grandes ${ }^{12}$.

Las características de la productividad de las instituciones son diferentes a las de los autores. Por un lado, presentan una mayor productividad individual, pues dos instituciones alcanzan un máximo de 12 trabajos, frente a los 7 por parte de 2 autores. Por otro, en conjunto las instituciones alcanzan una mayor productividad, ya que el $2,6 \%$ pueden clasificarse en el grupo de las grandes productoras (entre los autores no había grandes productores), siendo también superior el porcentaje de las que son medianas productoras (26\%, frente al 15,2\% de los autores). Ello viene a mostrar que entre las instituciones de procedencia de los autores existe una participación en los originales de la RESP más constante, que se refleja en el hecho de que el $28,6 \%$ de ellas ha participado en más de un trabajo (frente a un 15,2\% de los autores). No obstante, esta mayor productividad puede derivarse del hecho de que las instituciones máximas productoras son de ámbito nacional o autonómico, por lo que podrían albergar un elevado número de investigado- 
res, como el Instituto de Salud Carlos III o la Universidad.

Aunque en la distribución de los autores por sexo predomina el masculino, la diferencia entre hombres y mujeres es menor que en otras revistas $^{10,11,13,14}$, debido probablemente a que estos trabajos estudian sólo el sexo del primer autor, y en el nuestro se estudia a todos los autores que firman cada trabajo. En el artículo de Shiaffino referido al año 1998, la RESP ya mostraba una distribución más favorable de los autores hacia el sexo femenino $(44,7 \%)$ que las restantes revistas (Atención Primaria, 31,3\%; Gaceta Sanitaria, $34,4 \%$ o Medicina Clínica, 24\%) ${ }^{13}$. Para el período estudiado, la RESP muestra una cifra destacada en lo que se refiere al papel de las mujeres como primeras autoras ya que lo son al menos el 31,4\%, no habiendo sido posible contabilizar los trabajos en los que solo figura la inicial del nombre de pila. En el estudio de Yankauer sobre la revista American Journal of Public Health ${ }^{15}$, la cifra de mujeres como primeras autoras de los trabajos publicados fue creciendo desde el $3 \%$ en 1930 , al $30 \%$ en 1983, porcentaje cercano al de la RESP en la década estudiada.

Un estudio de JAMA $^{13}$, que investiga la proporción de mujeres entre los directores y los revisores de las revistas, además de la de los autores, concluye que el resultado es sobre todo muy bajo para las directoras $(12,8 \%)$, y algo más elevado para las revisoras $(26,7 \%)$ y las autoras $(28,7 \%)$, lo que puede derivarse de un posible sesgo de selección que favorece a los hombres. Así mismo, detecta que la proporción de primeras autoras aumenta cuando el tipo de trabajo se refiere a artículos originales, disminuyendo en el resto (editoriales, cartas al director o revisiones). En el periodo estudiado para la RESP hubo un porcentaje de evaluadoras del 35,2 \% (de un total de 443 evaluadores que revisaron trabajos para la revis$\left.\mathrm{ta}^{16}\right)$. Así pues, el porcentaje en la RESP de las mujeres resulta inferior al de los hombres, pero superior tanto para las primeras autoras de los originales como para el conjunto de las autoras en relación con otras revistas.

La distribución de instituciones por CCAA sitúa en primer lugar a las mismas Comunidades que aparecían destacadas en la primera parte del estudio ${ }^{1}$ : Comunidad de Madrid, Comunidad Valenciana, Andalucía y Cataluña. Éstas son las mismas que suelen figurar en la mayoría de los trabajos que estudian la producción científica española desde el punto de vista geográfico ${ }^{5,7,8,12,17}$. Como ya se comentó en la primera parte del trabajo, la presencia de estas Comunidades puede deberse a varios hechos, como contar con mayor número de instituciones, tener más recursos financieros o simplemente mayor peso demográfico en el conjunto del país. Sin embargo, en la distribución de los autores por origen geográfico en el presente estudio sobre la RESP, Castilla y León aparece en cuarto lugar, al igual que en el estudio de Mapfre $^{10}$, por delante de Cataluña que ocupa el quinto, lo que podría explicarse por la posible mayor implantación que tendrían en esta última Comunidad Autónoma otras revistas que también tratan aspectos de epidemiología y salud pública.

La presencia de casi un 5\% de autores extranjeros en los originales publicados por la RESP podría tener que ver con la repercusión que tiene el hecho de que cada vez más autores españoles tienden a publicar los resultados de sus trabajos en revistas anglosajonas, lo que deja un vacío que a su vez tiende a llenarse por otros autores extranje$\operatorname{ros}^{17}$. Lógicamente, en la RESP los autores extranjeros más frecuentes proceden de países latinoamericanos y, por lo tanto, hispanohablantes.

La distribución de los autores por tipo de institución en la que prestan servicios indica que la situación más frecuente es la de aquellos que trabajan en los centros de atención sanitaria pública, sobre todo los que se dedican a la atención especializada, lo que coin- 
cide con los resultados de otros estudios centrados también en publicaciones específicas $^{5,10}$, excepto con el de Yankauer, que muestra que los profesionales de la Universidad sustituyeron a los de los centros sanitarios en $1980^{15}$.

Al estudiar la distribución de las instituciones por tipo de centro, se observa la importancia de los de atención sanitaria, principalmente los dedicados a la atención primaria, lo que resulta coherente con los resultados de la primera parte de este estudio, donde la atención primaria de salud es una de las materias más frecuentemente estudiadas $^{1}$. De hecho, la RESP se encuentra en el núcleo de Bradford para las revistas que publican sobre esta materia según un estudio sobre indicadores bibliométricos en atención primaria ${ }^{12}$. En otros trabajos, como el ya mencionado sobre epidemiología y salud pública ${ }^{7}$, las instituciones más prolíficas fueron también asistenciales, en este caso los hospitales, seguidas de la Universidad. Son datos que corroboran lo expuesto por López Piñero cuando se refiere a las aportaciones españolas en la literatura internacional, mostrando que aproximadamente el $80 \%$ de los trabajos proceden de la Universidad y de las instituciones de la Seguridad Social ${ }^{18}$. En un trabajo más reciente, también se observa cómo las instituciones sanitarias, sobre todo las de la sanidad pública, experimentan un intenso crecimiento como centros en los que trabajan los autores de los trabajos ${ }^{17}$.

En cuanto a la discrepancia entre el protagonismo de la atención especializada cuando consideramos autores a las personas, y la atención primaria, cuando consideramos la autoría de las instituciones, podría estar relacionada con una mayor colaboración al firmar trabajos entre los profesionales de la atención especializada que entre los de la atención primaria.

Como conclusión, se puede decir que la RESP es una publicación científica sobre salud pública implantada en todo el territorio español y en el ámbito hispanoamericano, y que la productividad de los autores que publican en la RESP, la de las instituciones donde trabajan y la de las Comunidades Autónomas de donde proceden, es similar a la de otras revistas y menor que cuando se estudian ámbitos temáticos y territoriales en más de una publicación. El dato más llamativo es el encontrado respecto al papel más relevante que en la RESP han tenido las mujeres como primeras autoras de los trabajos, y que Castilla y León sea Comunidad Autónoma de origen de los autores más frecuentemente que Cataluña.

\section{AGRADECIMIENTOS}

Los autores agradecen a Evaristo Jiménez-Contreras, de la Universidad de Granada, la lectura del manuscrito y las recomendaciones realizadas.

\section{BIBLIOGRAFÍA}

1. Pérez Andrés C, Estrada Lorenzo JM, Villar Álvarez F, Rebollo Rodríguez MJ. Estudio bibliométrico de los artículos originales de la Revista Española de Salud Pública (1991-2000). Parte Primera: indicadores generales. Rev Esp Salud Pública 2002; 76: 659-72.

2. López Piñero JM, Terrada ML. Los indicadores bibliométricos y la evaluación de la actividad médico-científica. (III) Los indicadores de producción, circulación y dispersión, consumo de la información y respercusión. Med Clin (Barc) 1992; 98: 142-8.

3. López López P. Bibliometría: la medida de la información. En: Manual de información y documentación. Madrid: Ediciones Pirámide; 1996. p. 488-512.

4. López Piñero JM, Terrada ML. Los indicadores biliométricos y la evaluación de la actividad médicocientífica. (IV) La aplicación de los indicadores. Med Clin (Barc) 1992; 98: 384-8.

5. López Cózar ED, Ruiz Pérez R, Jiménez Contreras E. Calidad editorial, difusión e indicadores bibliométricos de la Revista Española de Enfermedades Digestivas. Rev Esp Enferm Dig 1999; 91: 1-32. 
6. Simó Miñana J, Gaztambide Ganuza M, Latour Pérez J. Producción científica de los profesionales españoles de atención primaria (1990-1997). Un análisis bibliométrico a partir de Medline. Aten Primaria 1999; 23 (supl 1): 14-28.

7. Álvarez Solar M, López González ML, Cueto Espinar A. Indicadores bibliométricos, análisis temático y metodológico de la investigación publicada en España sobre epidemiología y salud pública (1988-1992). Med Clin (Barc) 1998; 111: 529-35.

8. Camí J, Fernández MT, Gómez Caridad I. La producción científica española en biomedicina y salud. Un estudio a través de Science Citation Index (1986-1989). Med Clin (Barc) 1993; 101: 721-31.

9. Parra Hidalgo P, Marset Campos P, Ramos García E, San Eustaquio Tudanca F. Cincuenta años de la Revista de Sanidad e Higiene Pública (19261975). Análisis bibliométrico de su producción científica. Rev San Hig Pública 1983; 57: 9691038 .

10. Valera Garrido JF, de la Gala Sánchez F. Análisis bibliométrico de la productividad científica en la revista Mapfre Medicina. Mapfre Med 2001; 12: 157-67.

11. López Muñoz F, Marín F, Boya J. Evaluación bibliométrica de la producción científica española en Neurociencia. Análisis de las publicaciones de difusión internacional durante el periodo 1984-1993. Rev Neurol (Barc) 1996; 24 (128): 417-26.
12. Álvarez Solar M, López González ML, Cueto Espinar A. Indicadores bibliométricos de la investigación en atención primaria (1988-1992). Aten Primaria 1996; 18: 229-36.

13. Dickersin K, Fredman L, Flegal KM, Scott JD, Crawley B. Is there a sex bias in choosing editors? Epidemiology journals as an example. JAMA 1998; 280(3): 260-4.

14. Schiaffino A, García M, Fernández E. Autoría y presentación de resultados según el género en cuatro revistas biomédicas españolas. Gac Sanit 2001; 15: 251-4.

15. Yankauer A. The American Journal of Public Health: 1957-1973. Am J Public Health 1979; 69: 1061-5.

16. Ministerio de Sanidad y Consumo. La Revista Española de Salud Pública en CDROM (1991-2000) Madrid: Ministerio de Sanidad y Consumo; 2001.

17. Jiménez-Contreras E, Faba C, de Moya Anegón F. El destino de las revistas científicas nacionales. El caso español a través de una muestra (1950-90). Rev Esp Doc Cient 2001; 24: 147-61.

18. López Piñero JM, Terrada ML. Los indicadores bibliométricos y la evaluación de la actividad médico-científica. (II) La comunicación científica en las distintas áreas de las ciencias médicas. Med Clin (Barc) 1992; 98: 101-6. 